\title{
Two-dimensional chromatography of complex polymers. 3. Full analysis of polystyrene-poly(methyl methacrylate) diblock copolymers
}

\author{
Harald Pasch $^{1}$ *, Kibret Mequanint ${ }^{1}$, Jörg Adrian $^{2}$ \\ ${ }^{1}$ Deutsches Kunststoff-Institut (German Institute for Polymers), Schlossgartenstr. 6, \\ 64289 Darmstadt, Germany; Fax +49-6151-292855; hpasch@dki.tu-darmstadt.de \\ ${ }^{2}$ BASF AG, Kunststofflaboratorium, ZKM/A - G 200, 67056 Ludwigshafen, Germany
}

(Received: January 29, 2002; published: February 20, 2002)

\begin{abstract}
Poly(styrene-block-methyl methacrylate)s were fully analyzed by liquid chromatography at the critical point of adsorption (LC-CC) and two-dimensional chromatography. Operating at chromatographic conditions corresponding to the critical points of the homopolymers polystyrene and poly(methyl methacrylate), the block lengths distributions for the different blocks of the block copolymers were determined quantitatively. Information on the amounts and molar mass distributions of homopolymers and coupling products that were identified in the samples as by-products was obtained by on-line coupled 2D chromatography. It was shown that a complete picture of the molecular heterogeneity of block copolymers can be obtained only when information from different chromatographic experiments is combined. Size exclusion chromatography alone is inappropriate for evaluating the molecular heterogeneity of such samples.
\end{abstract}

\section{Introduction}

Block copolymers are complex macromolecular systems that are characterized by distributions in molar mass (MMD) and chemical composition (CCD). Average molar masses and chemical compositions of block copolymers can be determined by a variety of different techniques. Chromatographic separation, however, is required in most cases for the determination of distribution functions. Size exclusion chromatography (SEC) is the standard technique for MMD analysis, while CCD can be determined by means of interaction chromatography [1]. It has been shown by us and others that the molar masses of individual blocks in diblock and triblock copolymers can be measured by liquid chromatography at the critical point of adsorption (LC-CC) [2-7]. Operating at critical conditions of one block of the copolymer, this block does not contribute to retention and behaves "chromatographically invisible" [8-10]. At the same time the other block elutes in the SEC mode and can be quantified accordingly.

To evaluate the two-dimensional parameter field of the composition and the molar mass distribution of such block copolymers, a combination of different chromatographic techniques can be used. Using these techniques and combining them with each other or with a selective detector, two-dimensional information on CCD and MMD can be obtained $[1,11,12]$. A fully automated two-dimensional chromatographic system was developed by Kilz et al. several years ago [13-15]. It consists of two chromatographs, one of which separates by chemical composition or functionality, 
while the other, a SEC instrument, separates subsequently by size. Via a storage loop system, fractions from the first separation step are automatically transferred into the second separation system.

During the last few years, a number of applications of automated 2D chromatography has been described, including the analyses of poly(alkylene oxide)s, polyesters and epoxy resins [16-20]. The analysis of the grafting product of butyl acrylate onto poly(styrene-block-butadiene) by 2D chromatography was described by Adrian et al. [21]. The characterization of products resulting from the grafting of methyl methacrylate onto EPDM has been carried out by Augenstein and Stickler [22] in the offline mode and by Siewing et al. using fully automated 2D chromatography [23].

The present paper describes the full analysis of polystyrene-poly(methyl methacrylate) diblock copolymers by on-line coupled 2D chromatography. In the first dimension, LC-CC is used to separate with regard to chemical composition. In the second dimension, SEC provides information on molar mass distribution.

\section{Results and discussion}

Block copolymers are generally prepared by different techniques of living polymerization. Initially, anionic polymerization was the only technique to prepare well defined narrow disperse block copolymers. Meanwhile, other methods have been developed, including atom-transfer radical polymerization (ATRP) and reversible addition-fragmentation chain transfer polymerization (RAFT). It is generally accepted and demonstrated by SEC measurements that all these techniques yield block copolymers with narrow molar mass distributions. Less has been done to present evidence that the block copolymers are also uniform with regard to chemical composition.

In general it must be assumed that depending on the preparation procedure, in addition to the block copolymers, homopolymers and coupling products are formed due to different chain termination and chain transfer reactions. As has been shown previously [5,6], LC-CC can be used to separate block copolymers with regard to chemical composition. As a result, homopolymers are fractionated from the block copolymers and can be determined quantitatively. For the polystyrene-poly(methyl methacrylate) block copolymers (PS-b-PMMA) under investigation, such separations must be conducted under chromatographic conditions corresponding to the critical points of PS and PMMA.

In previous studies it has been shown that the critical point of PMMA can be obtained on a silica stationary phase with a mobile phase of methyl ethyl ketone-cyclohexane [5]. The critical point of PS corresponds to a chromatographic system comprising $\mathrm{C}_{18^{-}}$ modified silica as the stationary phase and tetrahydrofuran-acetonitrile as the mobile phase [6]. These experimental conditions are used to separate the PS-b-PMMA samples according to chemical composition.

As a first representative example, block copolymer 1 with a total molar mass of roughly $180000 \mathrm{~g} / \mathrm{mol}$ and an average chemical composition of PS:PMMA of 30:70 wt.-\% prepared by anionic polymerization is analyzed. The chromatograms of this sample taken in the SEC mode, at critical conditions for PS and critical conditions for PMMA, are presented in Fig. 1. As can be expected, SEC gives a uniform elution profile and narrow polydispersity. The chromatogram does not indicate any higher or lower molar mass by-products or chemically different species, see Fig. 1A. 

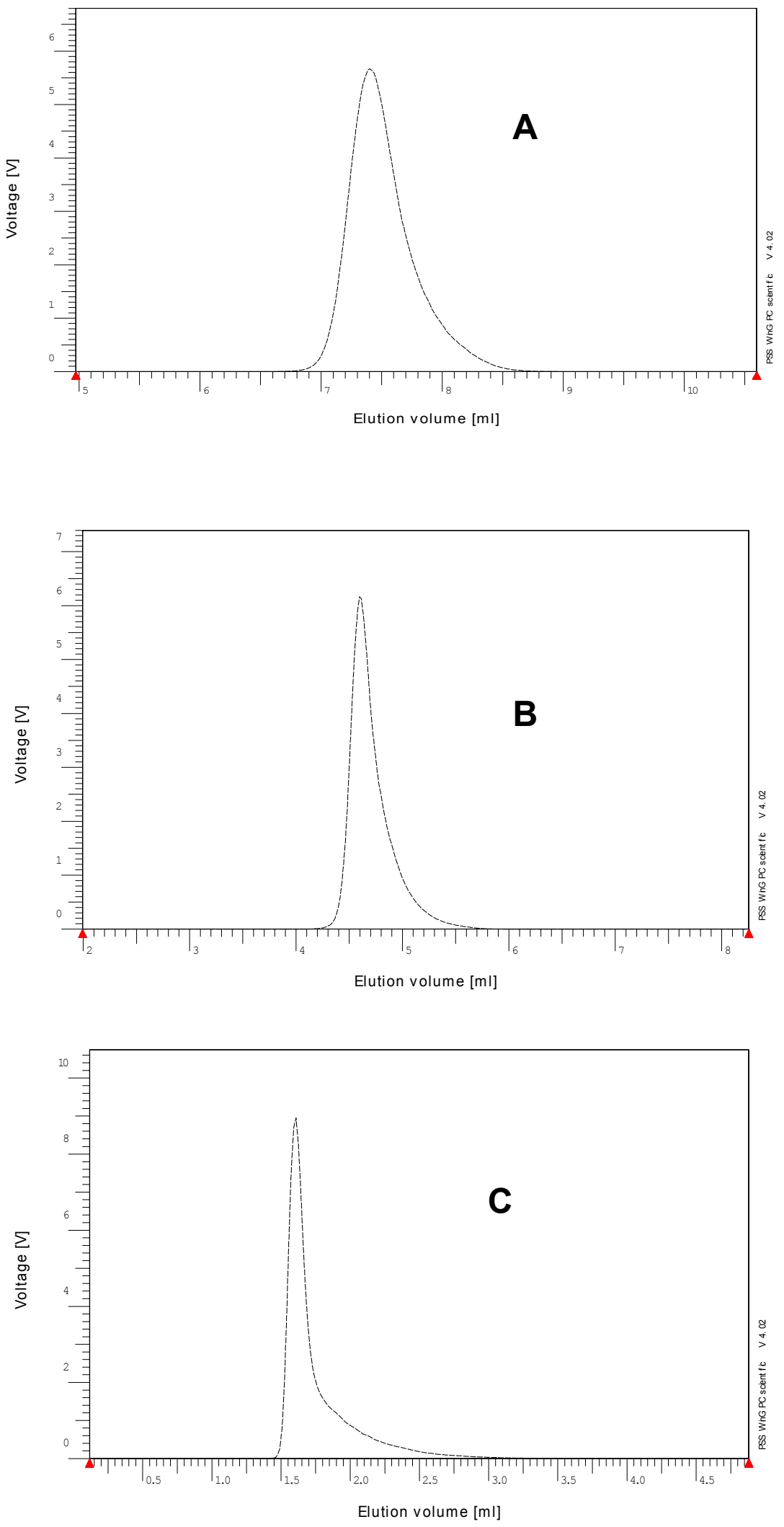

Fig. 1. SEC (A) and LC-CC chromatograms of PS-b-PMMA block copolymer 1, at critical conditions for PS (B) and PMMA (C), respectively; detector in all cases evaporative light scattering detector (ELSD) 
Different from the SEC elution profile, the LC-CC chromatogram taken at critical conditions for PS in Fig. 1B yields information on chemically different fractions. Keeping in mind that under these conditions the elution behaviour of the sample is solely directed by the PMMA block irrespective of the PS block, the elution profile represents the length distribution of this block. As has been shown previously, from such type of separation the block length of the PMMA block in the PS-b-PMMA block copolymer can be determined quantitatively by calibrating the system with PMMA calibration standards. The calculated molar mass values for the PMMA block are $\mathrm{M}_{\mathrm{w}}$ $=113000 \mathrm{~g} / \mathrm{mol}, M_{n}=97000 \mathrm{~g} / \mathrm{mol}$, and $M_{w} / M_{n}=1.16$. These data are in agreement with the expected values and the narrow polydispersity that can be assumed for an anionically polymerized sample.

More detailed information on the chemical composition of the block copolymer with regard to the PS block can be obtained by running a similar chromatographic experiment at the critical point for PMMA. Under these conditions the block copolymer is separated with regard to the PS block irrespective of the PMMA block. The chromatogram of this type of separation in Fig. 1C is rather uniform but with a slight tailing which might be due to a minute deviation from the critical point. Through a conventional calibration with PS standards, $M_{w}$ of roughly $70000 \mathrm{~g} / \mathrm{mol}$ is obtained for the PS block which again is in good agreement with the expected value. It has to be mentioned that the present chromatogram does not show any further fractions that could be an indication of homopolymers or coupling products.

On-line coupled two-dimensional (2D) chromatography combines methods that are separating into diverging directions of molecular heterogeneity. In general, one method separates according to chemical composition or functionality while another method is selective towards molar mass. As has been shown in previous applications, the resolving power and peak capacity of 2D chromatography is much higher than the sum of the single dimensions [24]. Therefore, 2D separations of the block copolymer are carried out in the following experiments using LC-CC in the first and SEC in the second dimension. In order to resolve all peculiarities of the sample, experiments at both critical points are conducted.

The contour diagrams of the 2D experiments conducted at critical conditions for PS (A) and PMMA (B) are presented in Fig. 2. The ordinate represents the separation in the first dimension, while the abscissa indicates the SEC separation of the fractions. The molar mass calibration was carried out using PMMA (A) and PS (B) calibration standards, respectively. The generation of a contour diagram from the individual SEC chromatograms of the fractions is discussed in ref. [1]. The contour plots indicating very high homogeneity with regard to chemical composition and molar mass are in agreement with the results of the one-dimensional analyses where no indications of homopolymers and coupling products have been found. From the contour plots the molar mass of the block copolymer can be calculated. Based on a PMMA calibration the following molar masses are obtained from the contour plot in Fig. $2 A: M_{w}=$ $164000 \mathrm{~g} / \mathrm{mol}, M_{n}=150000 \mathrm{~g} / \mathrm{mol}$, and $M_{w} / M_{n}=1.09$. From the molar masses of the individual blocks that have been determined by LC-CC, a total molar mass of $\mathrm{M}_{\mathrm{w}}=$ $183000 \mathrm{~g} / \mathrm{mol}$ would be expected for the block copolymer. The deviation can be explained by the fact that for the quantification of the PS-b-PMMA block copolymer a PMMA calibration curve has been used. It is known that due to the different hydrodynamic volumes of PS and PMMA deviations from the true molar masses can be encountered for copolymers. Obviously, a molar mass sensitive detector, e.g. a light scattering detector or an on-line viscometer, would yield better molar mass data. 
However, for experimental reasons the coupling of 2D chromatography with these detectors is not possible yet.
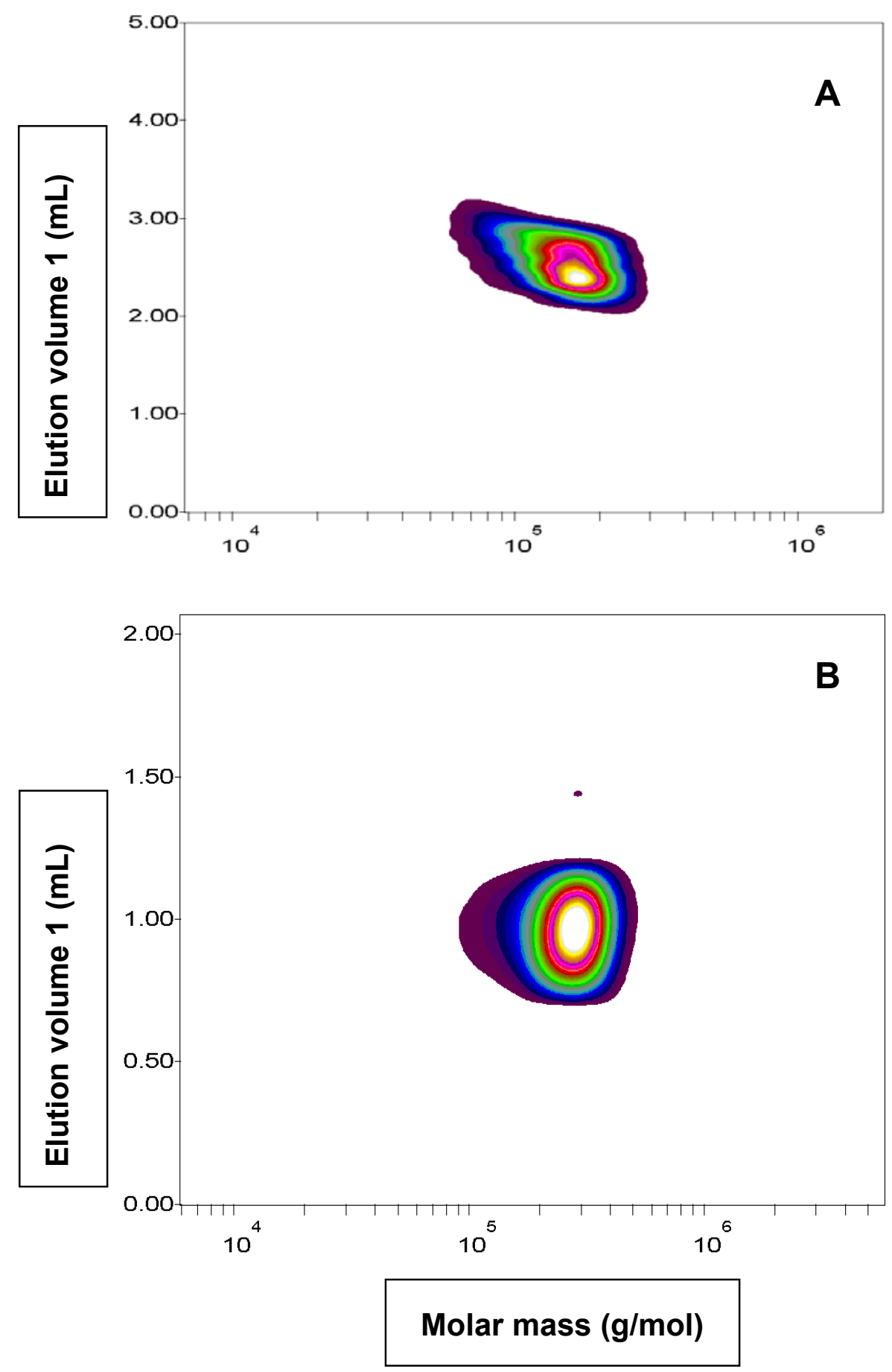

Fig. 2. Contour diagrams of 2D chromatography of PS-b-PMMA block copolymer 1; 1st dimension: critical conditions for PS (A) and PMMA (B), respectively, 2nd dimension: SEC; detector: ELSD

Very frequently, block copolymers are not as homogeneous as SEC suggests. In the following example, a PS-b-PMMA block copolymer (sample 2) of an average molar mass of $25000 \mathrm{~g} / \mathrm{mol}$ is analyzed by SEC and LC-CC at critical conditions for PS, see Fig. 3. While SEC indicates that the sample is narrow distributed with regard to 
molar mass, the LC-CC trace clearly shows a second component in addition to the diblock copolymer. From the elution volume and a comparison of the UV and ELSD traces, indicating higher UV activity as compared to the block copolymer, the component with an elution volume of $6.7 \mathrm{~mL}$ can be assigned to PS homopolymer. As is known, homopolymers are formed when chain termination takes place before the second monomer is added to the reaction mixture. Since polymerization was started with styrene, PS can be formed as the homopolymer.
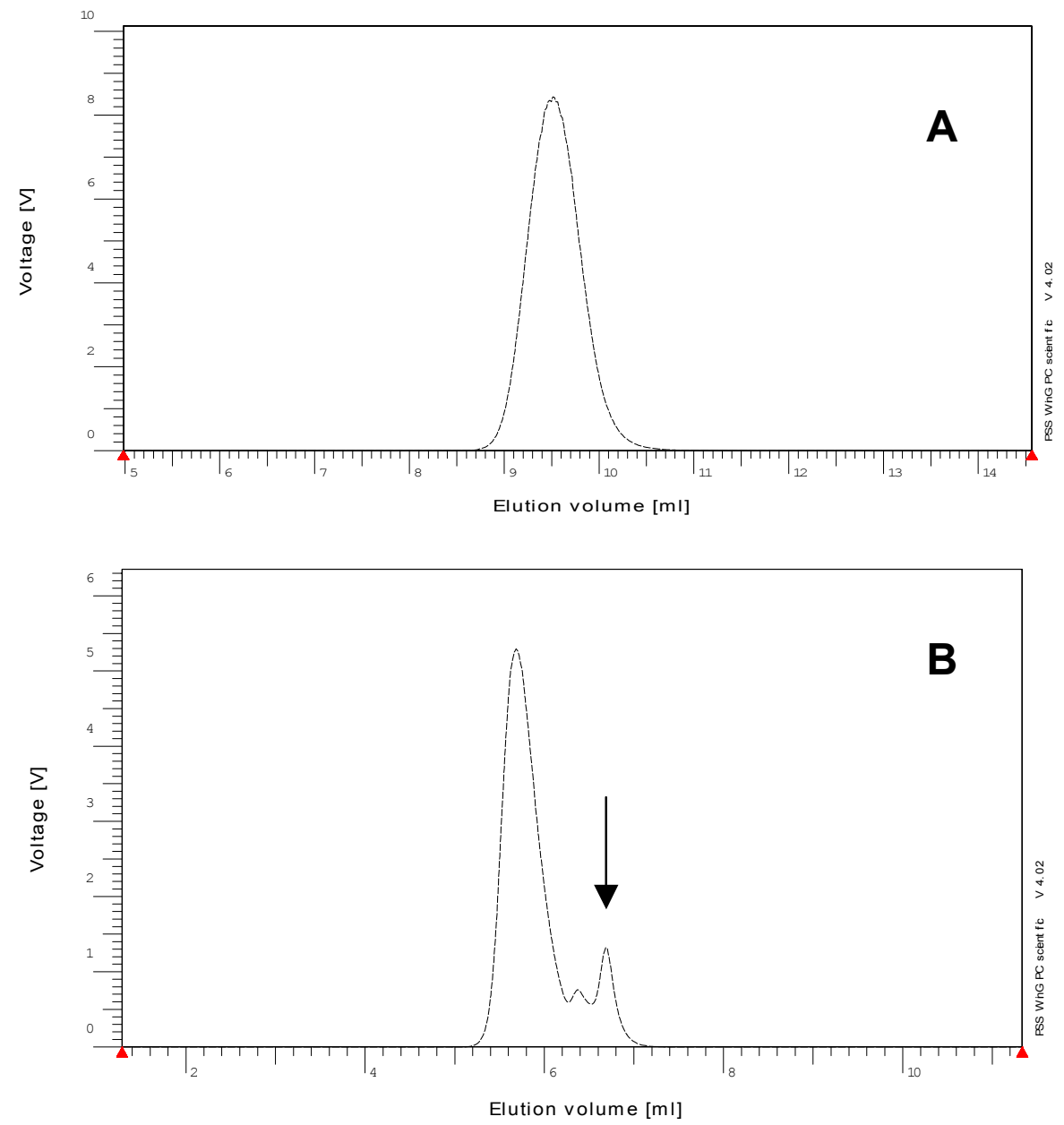

Fig. 3. SEC (A) and LC-CC (B) chromatograms of PS-b-PMMA block copolymer 2 , critical conditions for PS, detector: ELSD

Due to the fact that the LC-CC separation of the block copolymer and the PS homopolymer is not baseline-resolved, the calculated molar mass distribution of the PMMA block can only be tentative. It amounts to $M_{w}=12000 \mathrm{~g} / \mathrm{mol}$ and $M_{n}=9000$ $\mathrm{g} / \mathrm{mol}$.

A much better separation is obtained by $2 \mathrm{D}$ chromatography where the 1 st dimension corresponds to LC-CC conditions for PS, see Fig. 4. Accordingly, much more precise molar mass calculations can be conducted. The calibration of the molar mass axis is done with narrow disperse polystyrenes.

In addition to the molar masses, the relative amounts of the components can be determined. The data obtained from the 2D experiment are summarized in Tab. 1. From the molar masses of the block copolymer and the PMMA block, the molar mass 
of the PS block can be calculated to be roughly $M_{w}=13000 \mathrm{~g} / \mathrm{mol}$ and $M_{n}=12000$ $\mathrm{g} / \mathrm{mol}$.

As is shown in Fig. 4, in addition to the diblock copolymer and the PS homopolymer, a third component is present in the sample. The molar mass of this component is higher than the molar mass of PS but lower than the molar mass of the block copolymer. At present, the nature of this polymeric component is unknown.

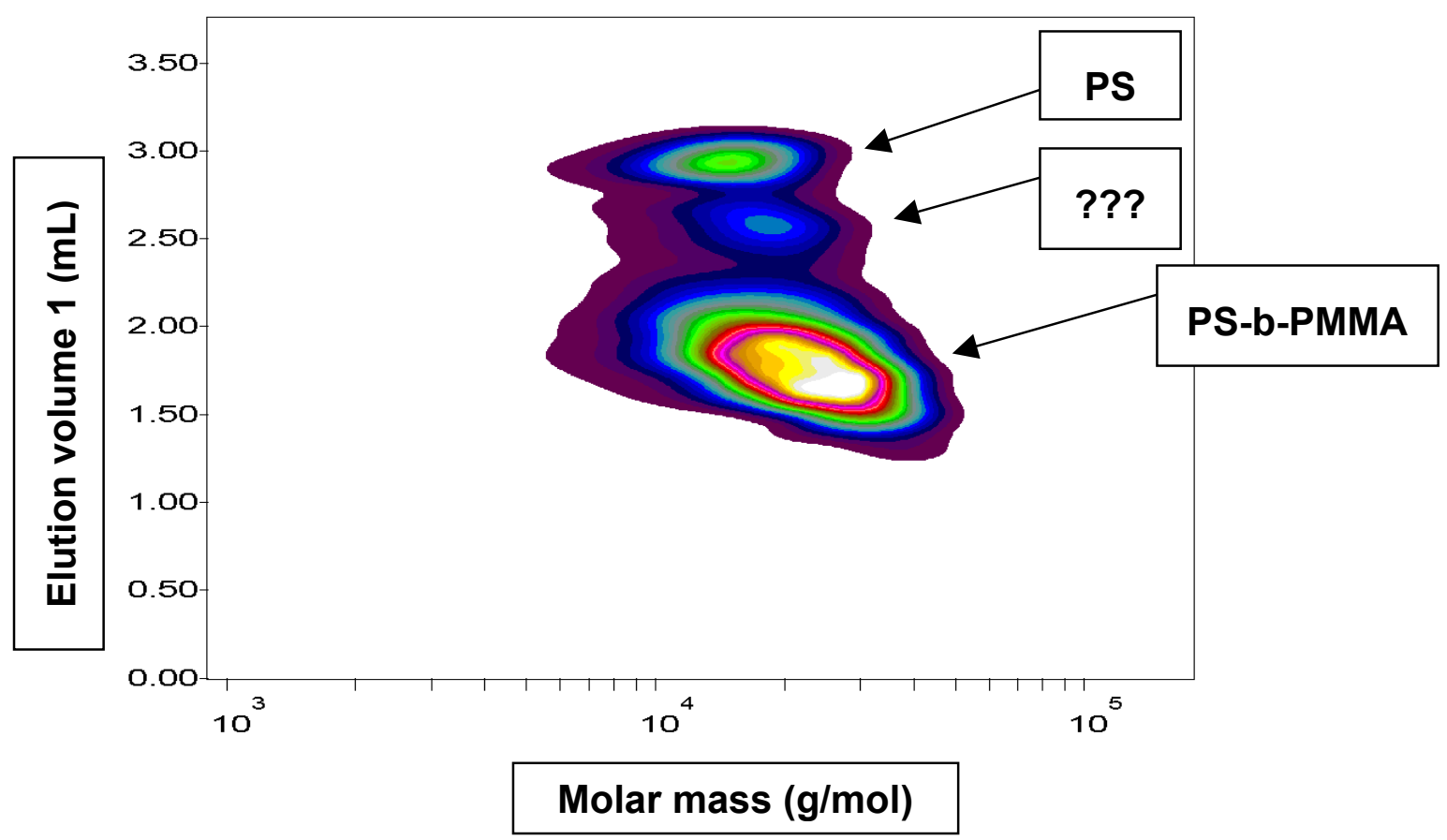

Fig. 4. Contour diagram of $2 \mathrm{D}$ chromatography of PS-b-PMMA block copolymer 2; 1st dimension: critical conditions for PS, 2nd dimension: SEC; detector: ELSD

Tab. 1. Analytical data for PS-b-PMMA block copolymer 2, obtained by 2D chromatography; "Total" results from the integration of the total contour plot area

\begin{tabular}{|l|r|r|r|r|}
\hline & $\begin{array}{c}\text { Relative } \\
\text { amount in \% }\end{array}$ & $M_{\mathrm{w}}$ in $\mathrm{g} / \mathrm{mol}$ & $\mathrm{M}_{\mathrm{n}}$ in $\mathrm{g} / \mathrm{mol}$ & $\mathrm{M}_{\mathrm{w}} / \mathrm{M}_{\mathrm{n}}$ \\
\hline PS homopolymer & $13 \%$ & 15000 & 13000 & 1.15 \\
Fraction '???' & $14 \%$ & 18500 & 15700 & 1.18 \\
PS-b-PMMA & $73 \%$ & 25000 & 21000 & 1.19 \\
Total & $100 \%$ & 21500 & 19000 & 1.13 \\
\hline
\end{tabular}

As the next example a block copolymer with a total molar mass of $108000 \mathrm{~g} / \mathrm{mol}$ and an average chemical composition of PS:PMMA of 50:50 wt.-\% prepared by anionic polymerization is analyzed (sample 3 ). The chromatograms of this sample taken in the SEC mode, at critical conditions for PS and critical conditions for PMMA, are presented in Fig. 5. Similar to the previous samples, SEC gives a uniform elution profile indicating narrow polydispersity, see Fig. 5A. 

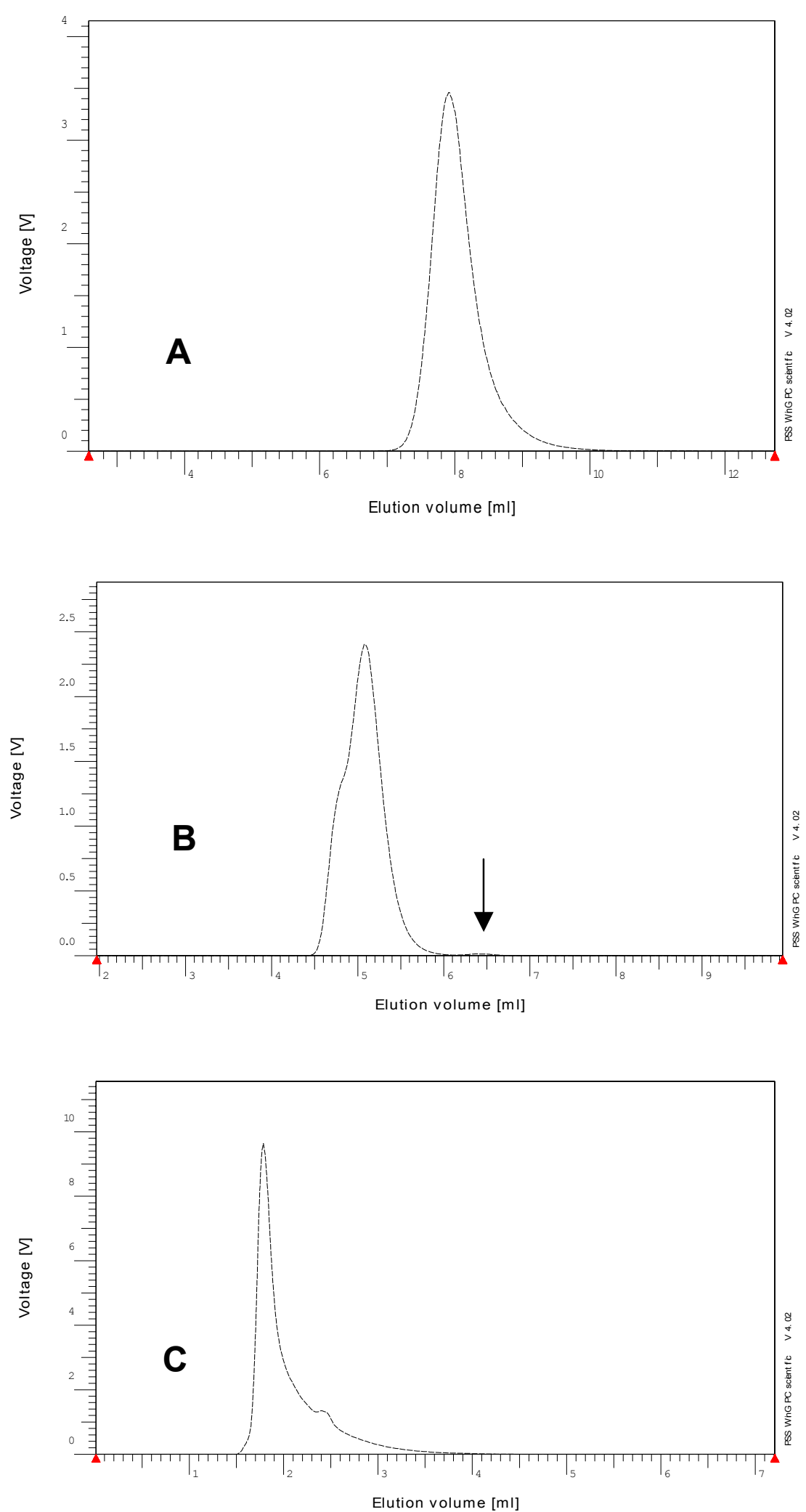

Fig. 5. SEC (A) and LC-CC chromatograms of PS-b-PMMA block copolymer 3; critical conditions for PS (B) and PMMA (C); detector in all cases ELSD

Different from the SEC elution profile, the LC-CC chromatogram taken at critical conditions for PS in Fig. 5B indicates chemically different fractions. Keeping in mind that under these conditions the elution behaviour of the sample is solely directed by the PMMA block irrespective of the PS block, the small fraction appearing at an 
elution volume of $6.4 \mathrm{~mL}$ (indicated by arrow) can be assigned to PS homopolymer. This has been verified further by analyzing this fraction by FTIR spectroscopy.

The main elution peak exhibits a shoulder at lower retention volumes indicating a fraction that, with regard to the PMMA block, has a higher molar mass than the main elution peak centered around an elution volume of $5.1 \mathrm{~mL}$. As has been shown previously, from such type of separation the block length of the PMMA block in the PS-b-PMMA block copolymer can be determined by calibrating the system with PMMA calibration standards. The peak maximum molar mass $\mathrm{M}_{\mathrm{p}}$ for the main elution peak is $54000 \mathrm{~g} / \mathrm{mol}$, which is in agreement with the expected value given in Tab. 3 . For the higher molar mass shoulder, a $M_{p}$ of $80000 \mathrm{~g} / \mathrm{mol}$ is obtained indicating a fraction having a PMMA block with a molar mass that is significantly larger than the original PMMA block.

As it is known that recombination can take place during the polymerization process, this fraction is tentatively assigned to a coupling product. Unfortunately, due to incomplete separation only the total molar mass distribution of the PMMA block including the coupling product can be determined giving $M_{w}=59000 \mathrm{~g} / \mathrm{mol}, M_{n}=$ $47000 \mathrm{~g} / \mathrm{mol}$ and a polydispersity of $\mathrm{M}_{\mathrm{w}} / \mathrm{M}_{\mathrm{n}}=1.25$.

More detailed information on the chemical composition of the block copolymer with regard to the PS block can be obtained by running a similar chromatographic experiment at the critical point for PMMA. Under these conditions the block copolymer is separated with regard to the PS block irrespective of the PMMA block. The chromatogram of this type of separation in Fig. $5 \mathrm{C}$ is rather uniform but with a slight tailing. Through a conventional calibration with PS standards a $M_{p}$ value of $58000 \mathrm{~g} / \mathrm{mol}$ is obtained for the PS block which again is in good agreement with the expected number. It has to be mentioned that the present chromatogram does not show any higher molar mass shoulders that could be an indication of coupling products.

The contour diagrams of the 2D experiments conducted at critical conditions for $\mathrm{PS}$ (A) and PMMA (B) are presented in Fig. 6. Comparing the 2D plot in Fig. 6A with the LC-CC chromatogram in Fig. 5B it is obvious that resolution is significantly improved. The molar mass calibration was carried out using PMMA $(A)$ and PS (B) calibration standards. The contour plot $(A)$ indicates three fractions which are different in chemical composition and molar mass. The assignment of the fractions is straightforward and based on the LC-CC separation. They correspond to PS, PS-g-PMMA, and the coupling product, respectively.

The contour plot clearly indicates that the molar masses of the three components overlap. Therefore, obviously, an SEC type separation alone (as in Fig. 5A) cannot resolve the different components of the block copolymer. The relative concentrations of the components are obtained from the intensities of the contour plot peaks. For sample 3, PS-g-PMMA has the highest concentration, while the concentration of the coupling product is lower, see Fig. 6A. The contour plot taken at critical conditions for PMMA in Fig. 6B shows that the sample does not contain residues of PMMA homopolymer. 

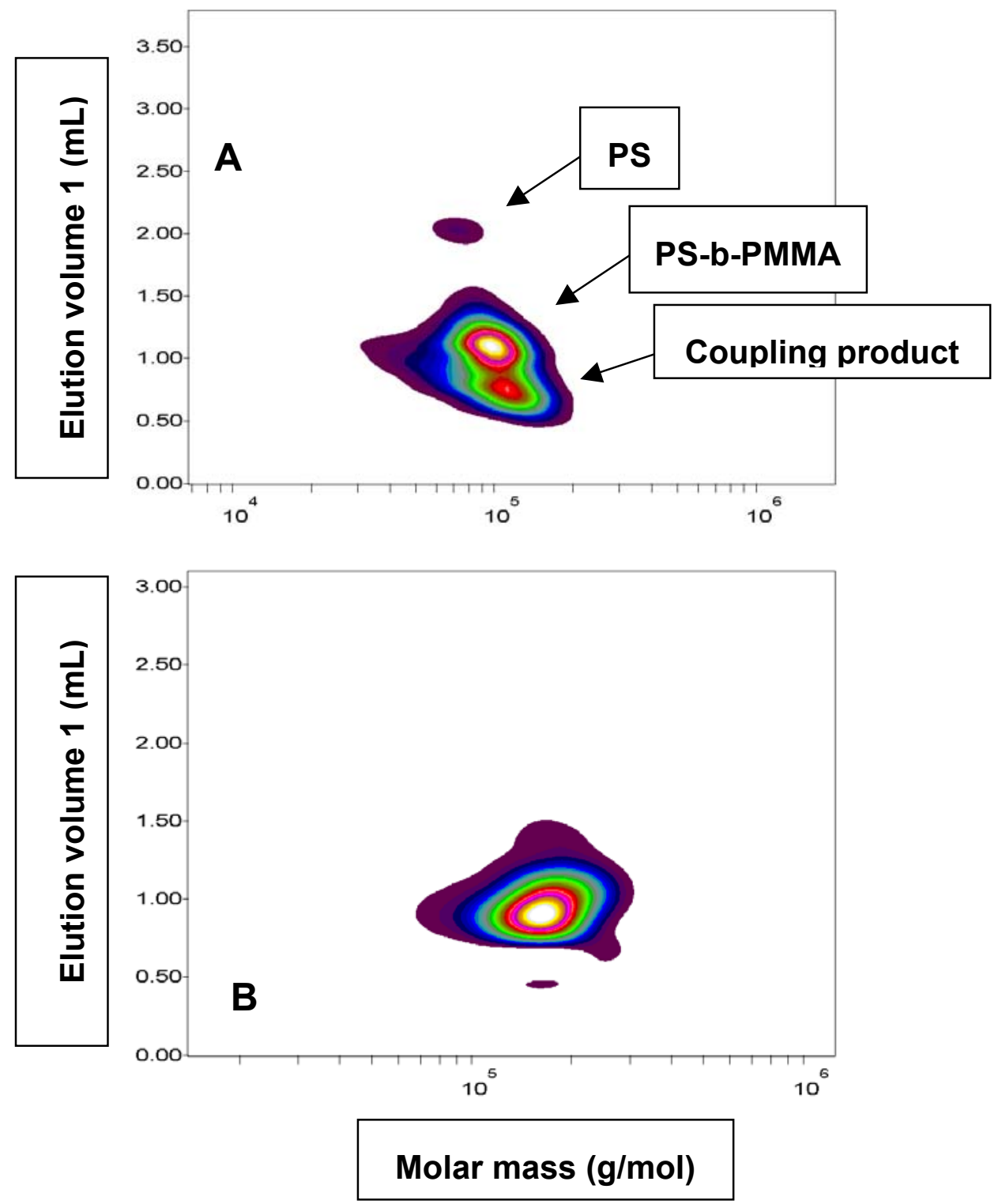

Fig. 6. Contour diagrams of 2D chromatography of PS-b-PMMA block copolymer 3; 1st dimension: critical conditions for PS (A) and PMMA (B), respectively, 2nd dimension: SEC; detector: ELSD

Comparing the total molar mass of the block copolymer taken from 2D chromatography $\left(M_{w}=95900 \mathrm{~g} / \mathrm{mol}\right)$ with the sum of the PS and PMMA blocks taken from LC-CC $\left(M_{w}=58000 \mathrm{~g} / \mathrm{mol}+54000 \mathrm{~g} / \mathrm{mol}=112000 \mathrm{~g} / \mathrm{mol}\right)$, the $2 \mathrm{D}-\mathrm{LC}$ value is gradually too low. As has been explained already, this is due to the calibration of the 2D system with PMMA.

A summary of the compositions and the molar masses of the sample components is given in Tab. 2. 
Tab. 2. Analytical data for PS-b-PMMA block copolymer 3, obtained by LC-CC and 2D chromatography

\begin{tabular}{|c|c|c|c|c|}
\hline & $M_{p}$ in $\mathrm{g} / \mathrm{mol}$ & $\mathrm{M}_{\mathrm{w}}$ in $\mathrm{g} / \mathrm{mol}$ & $M_{n}$ in $\mathrm{g} / \mathrm{mol}$ & $M_{w} / M_{n}$ \\
\hline \multicolumn{5}{|l|}{ LC-CC ${ }^{1}$} \\
\hline $\begin{array}{l}\text { PMMA block of diblock } \\
\text { copolymer }\end{array}$ & 54000 & & & \\
\hline $\begin{array}{l}\text { PMMA blocks of coupling } \\
\text { product }\end{array}$ & 80000 & & & \\
\hline total PMMA blocks & & 59000 & 46800 & 1.26 \\
\hline \multicolumn{5}{|l|}{$\mathrm{LC}-\mathrm{CC}^{2}$} \\
\hline $\begin{array}{l}\text { PS block of diblock } \\
\text { copolymer }\end{array}$ & 58000 & & & \\
\hline \multicolumn{5}{|l|}{ 2D chromatography ${ }^{1}$} \\
\hline 60.4\% Diblock copolymer & & 95900 & 88900 & 1.08 \\
\hline $35.4 \%$ Coupling product & & 124000 & 118000 & 1.05 \\
\hline 1.4\% PS homopolymer & & 77700 & 76300 & 1.02 \\
\hline
\end{tabular}

${ }^{1}$ Critical conditions for PS.

${ }^{2}$ Critical conditions for PMMA.

In order to show that SEC is completely inadequate for the evaluation of the molecular homogeneity of a block copolymer, the SEC and 2D chromatography plots for two more PS-b-PMMA block copolymers are presented in Fig. 7. While SEC again indicates narrow molar mass distribution, the 2D-LC plots clearly show that the samples are very heterogeneous with regard to chemical composition. These samples are far from being pure diblock copolymers.

For a comparison to samples prepared by anionic polymerization, the following section describes the analysis of block copolymers that have been synthesized by RAFT in the laboratory of E. Rizzardo. In this case the polymerization has been started with methyl methacrylate and, accordingly, PMMA-b-PS diblock copolymers were obtained. Fractions of the reaction mixtures were terminated after the first polymerization step and the corresponding PMMA block precursors were isolated. Two different molar masses of the PMMA block have been prepared, namely 20000 (6a) and $38000 \mathrm{~g} / \mathrm{mol}$ (7a). 

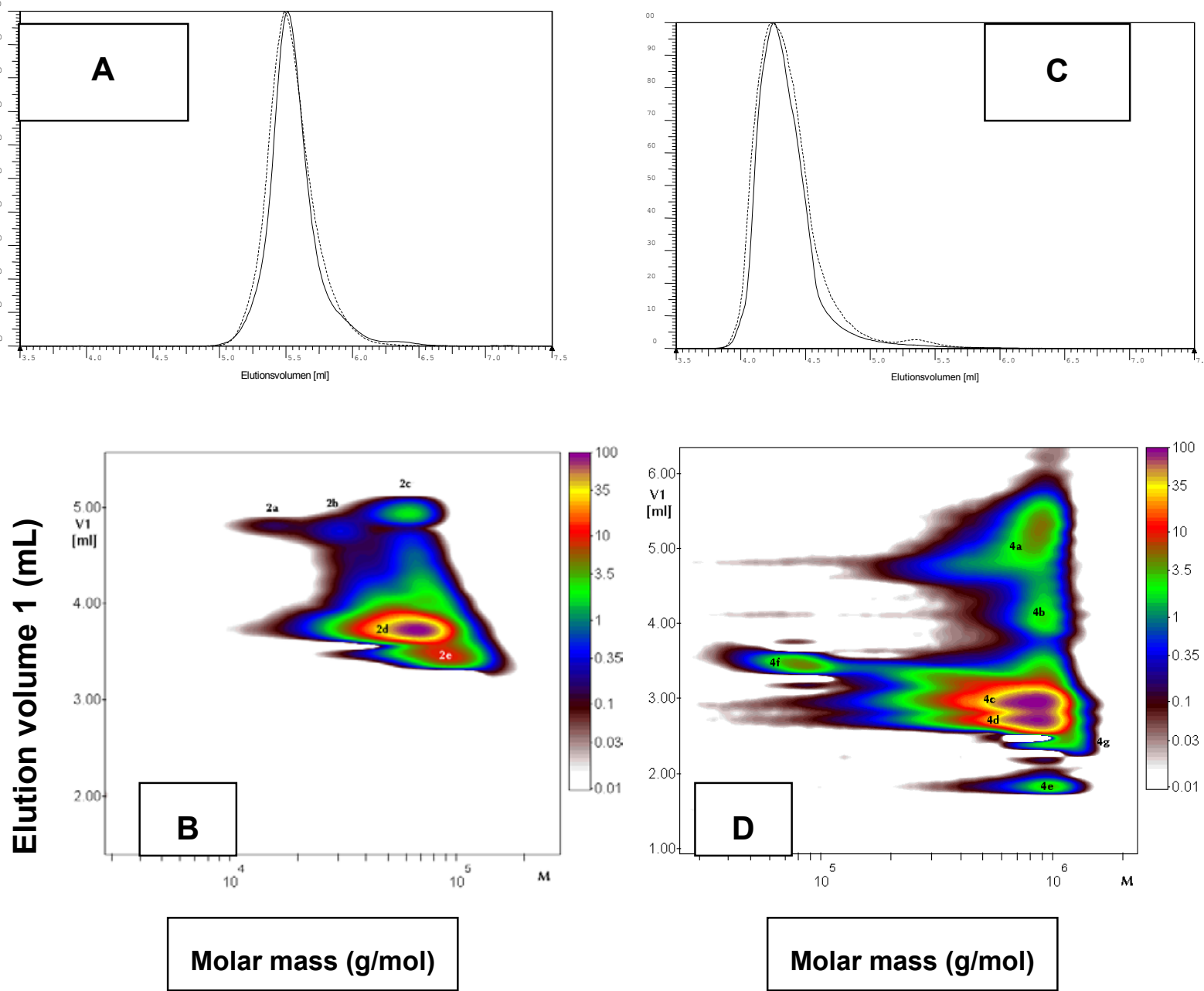

Fig. 7. SEC $(A, C)$ and $2 D-L C(B, D)$ of PS-b-PMMA block copolymers $4(A, B)$ and 5 (C,D); 1st dimension in 2D-LC: critical conditions for PS, 2nd dimension in 2D-LC: SEC; detectors: ELSD (outer curves) and UV $254 \mathrm{~nm}$

The analysis of the PMMA block precursors by SEC, LC-CC at critical conditions for $\mathrm{PS}$, and 2D chromatography shows clearly that the samples have narrow molar mass distributions and they are homogeneous with regard to chemical composition, see Fig. 8. In particular, the 2D plots don't show any indications of by-products that are different from the precursors with regard to molar mass or composition. The molar mass distributions that are determined from the 2D plot are in very good agreement with the expected values:

$\begin{array}{llll}\text { 6a: } & M_{w}=22000 \mathrm{~g} / \mathrm{mol} & M_{n}=18900 \mathrm{~g} / \mathrm{mol} & M_{w} / M_{n}=1.16 \\ \text { 7a: } & M_{w}=39400 \mathrm{~g} / \mathrm{mol} & M_{n}=35800 \mathrm{~g} / \mathrm{mol} & M_{w} / M_{n}=1.10\end{array}$



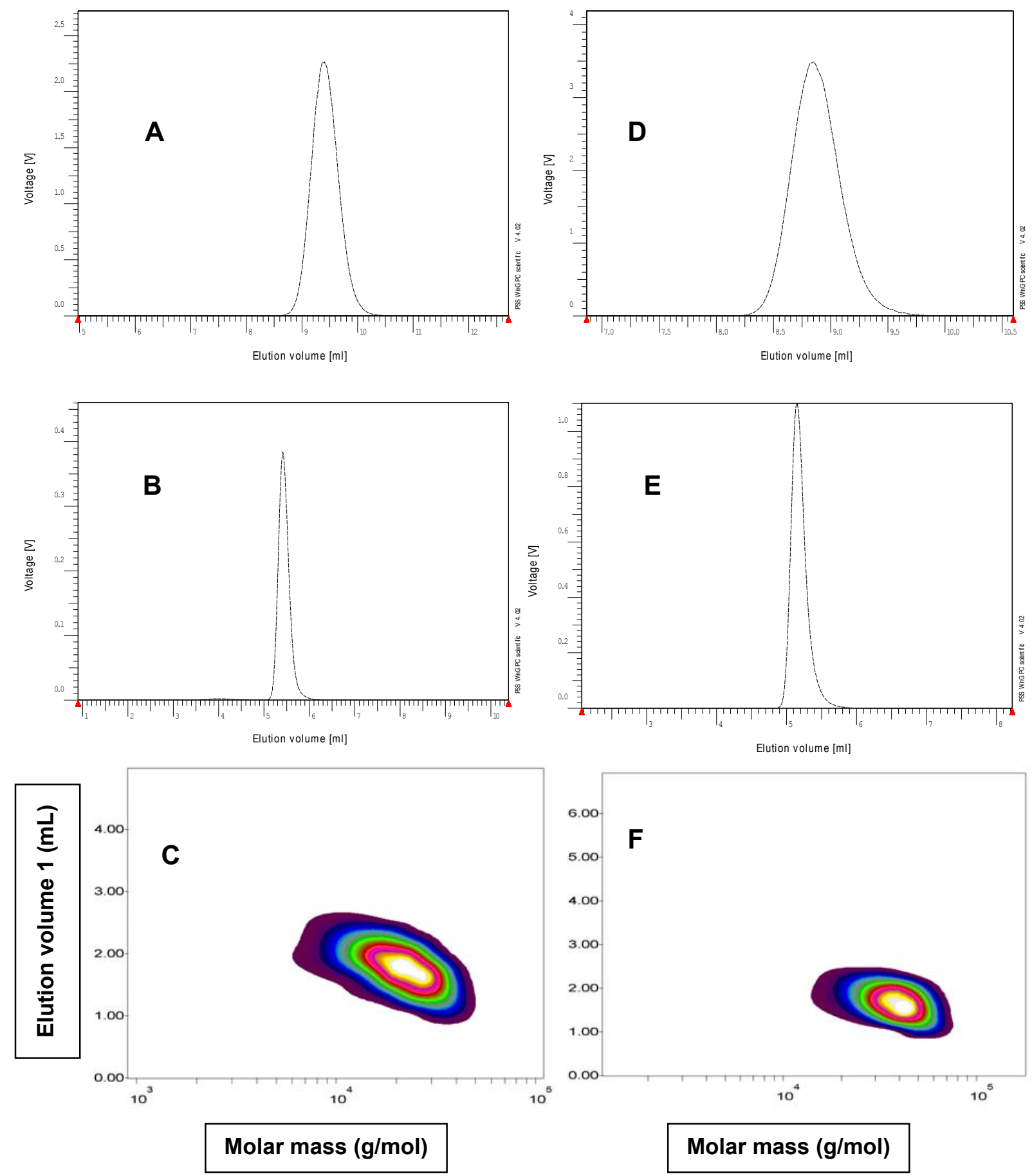

Fig. 8. SEC $(A, D), L C-C C$ at critical conditions for $P S(B, E)$ and $2 D-L C(C, F)$ of PMMA block precursors $6 a(A, B, C)$ and $7 a(D, E, F)$, 1st dimension in 2D-LC: critical conditions for PS, 2nd dimension in 2D-LC: SEC; detector: ELSD

Subsequently, the PMMA-b-PS diblock copolymers that are prepared from the corresponding PMMA precursor blocks are analyzed by SEC, LC-CC and 2D chromatography. Similar to the previous investigations, LC-CC is conducted at critical conditions for both PMMA and PS. The SEC and LC-CC curves for the diblock copolymers $\mathbf{6}$ and $\mathbf{7}$ are summarized in Fig. 9. 

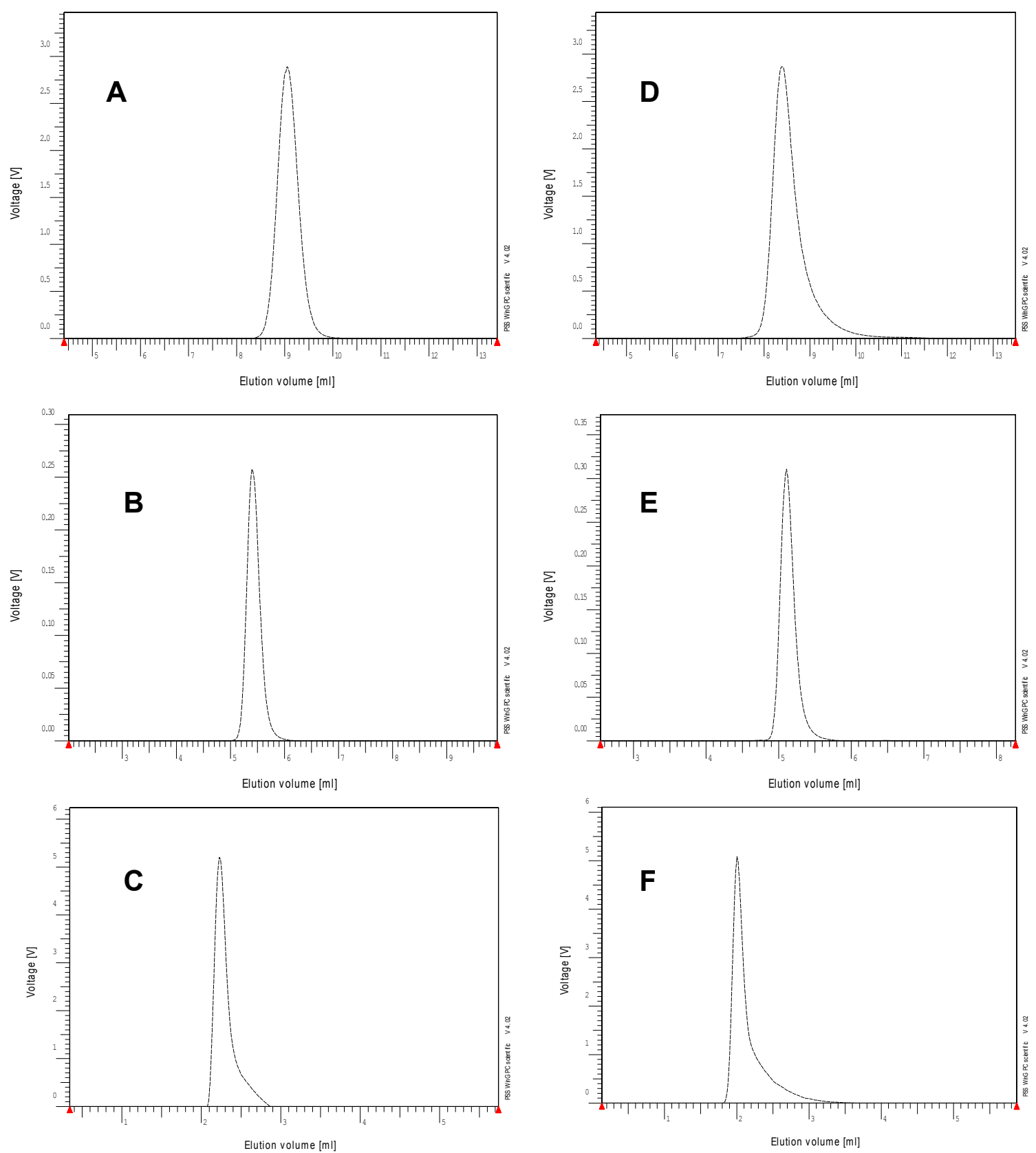

Fig. 9. SEC (A,D), and LC-CC chromatograms of diblock copolymers $6(A, B, C)$ and 7 $(D, E, F)$; critical conditions for PS $(B, E)$, critical conditions for PMMA (C,F); detector: ELSD

Similar to the PMMA precursors, the PMMA-b-PS copolymers appear to be very uniform with regard to both molar mass and chemical composition. The SEC and LC-CC elution curves exhibit only one uniform peak. There are no indications of the presence of homopolymers or coupling products. To verify these results, $2 \mathrm{D}$ experiments were carried out using both PS and PMMA critical conditions in the first dimension. The resulting contur plots for these experiments are presented in Fig. 10. 

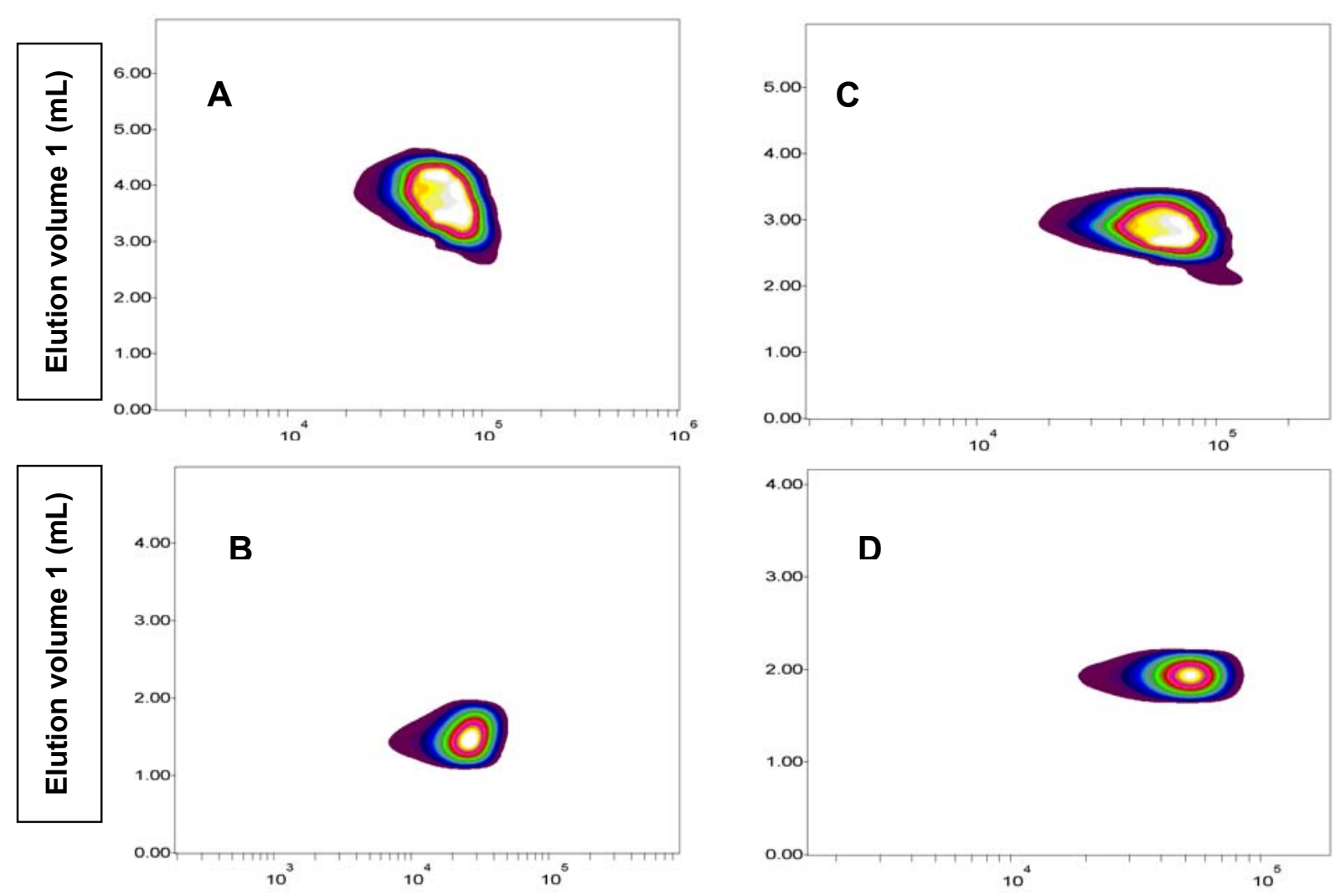

Molar mass (g/mol)

Molar mass (g/mol)

Fig. 10. 2D-LC analysis of diblock copolymers $6(A, B)$ and $7(C, D) ; 1$ st dimension: critical conditions for PS $(A, C)$ and PMMA (B,D); 2nd dimension: SEC; detector: ELSD

The 2D experiments confirm the remarkable molar mass and compositional homogeneity of the block copolymers.

Finally, the chromatographic behaviour of the block copolymers and the corresponding PMMA block precursors under LC-CC conditions shall be compared. According to the theory, at critical conditions for polystyrene the PS block of the diblock copolymers shall not contribute to retention. Therefore, the elution behaviour of the precursors and the block copolymers are expected to be identical. The elution curves of the PMMA-b-PS block copolymers and the corresponding PMMA block precursors at LC-CC conditions for polystyrene are presented in Fig. 11. As can be seen, they are very similar with regard to peak position and peak shape. This is a further proof for the "invisibility concept" of LC-CC.

The molar masses of the PS blocks are determined using LC-CC conditions corresponding to the critical point of PMMA. These measurements yield $M_{p}(P S)=9000$ $\mathrm{g} / \mathrm{mol}$ and $M_{p}(P S)=21500 \mathrm{~g} / \mathrm{mol}$ for samples 6 and 7 , respectively. From the PMMA-block and PS-block molar masses, the total molar masses of the block copolymers can be calculated to be roughly $21000 \mathrm{~g} / \mathrm{mol}$ and $60900 \mathrm{~g} / \mathrm{mol}$ for samples 6 and 7 , being in good agreement with the producers data. 

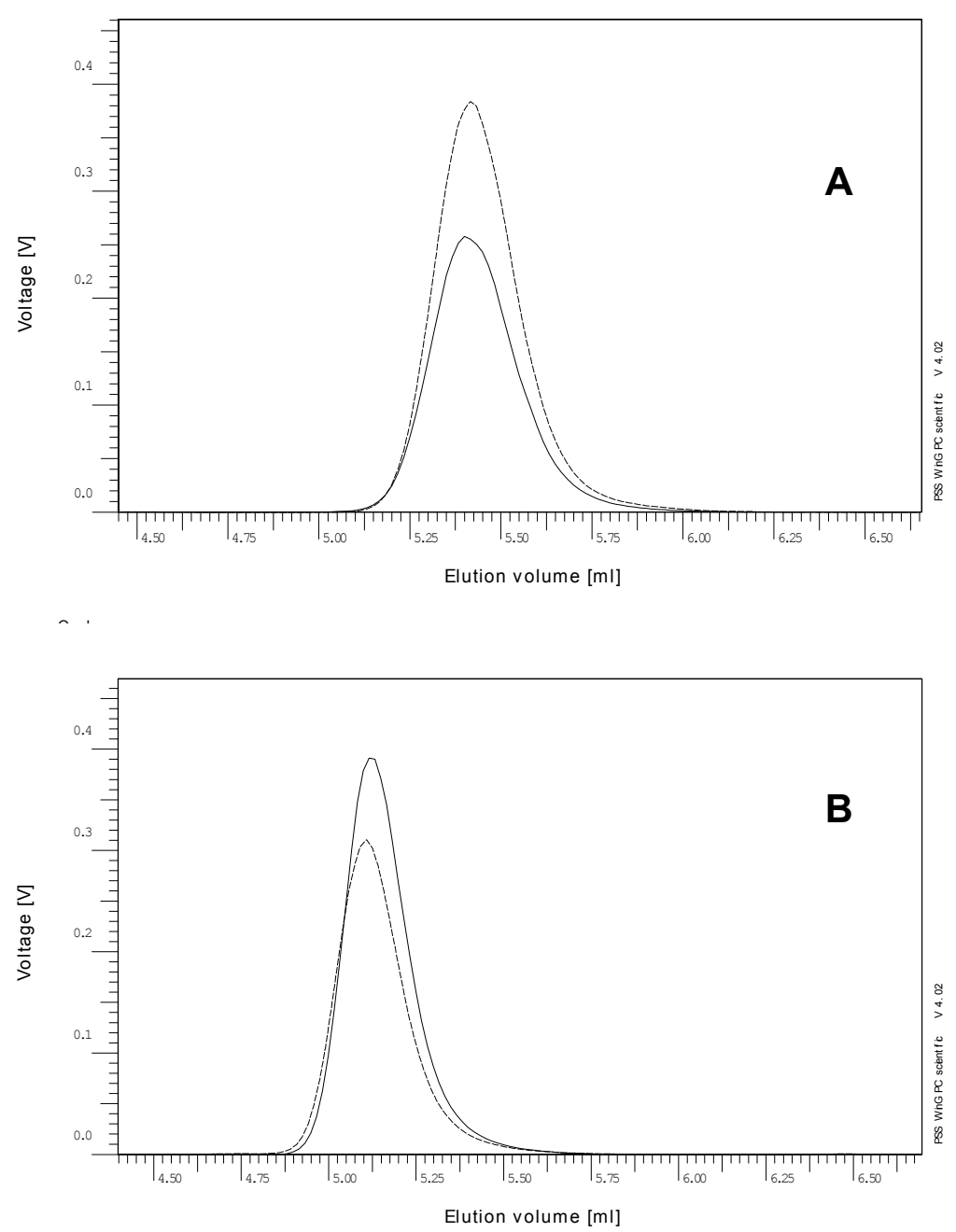

Fig. 11. LC-CC elution curves of the diblock copolymers 6 and 7 (upper curve in A, lower curve in $\mathrm{B}$ ) and the corresponding PMMA block precursors $6 \mathbf{a}$ and $7 \mathrm{a}$; critical conditions for PS; detector: ELSD; A: samples $\mathbf{6}$ and $\mathbf{6 a}$, B: samples 7 and $7 \mathbf{7 a}$

\section{Experimental part}

\section{Chromatographic system}

A modular chromatographic system comprising two chromatographs connected via one eight-port injection valve and two storage loops was used. The chromatograph for the first separation step (chromatograph 1) comprised a Rheodyne six-port injection valve with a $100 \mu \mathrm{L}$ injection loop and a ISCO syringe pump Model 100D. One electrically driven eight-port injection valve (Valco EHC8W) was used to connect the two chromatographs. In addition, they were connected to two storage loops of a volume of $100 \mu \mathrm{L}$ each. The chromatograph for the second separation step (chromatograph 2) comprised a Waters model 510 pump. The operation of the coupled injection valves was controlled by the software, which was used for data collection and processing. In the present case the software package "PSS-2D-GPC-Software" of Polymer Standards Service, Mainz, Germany, was used. Molar mass calibration is based on polystyrene and poly(methyl methacrylate). 


\section{Columns}

Chromatograph 1: (a) critical conditions for PS: Macherey\&Nagel Nucleosil $\mathrm{C}_{18} 300-$ 5+1000-7 $\AA$, 5 and $7 \mu \mathrm{m}$ average particle sizes. Column size was $250 \times 4 \mathrm{~mm}$ i.d. (b) critical conditions for PMMA: Macherey\&Nagel Nucleosil Si-300-5 A, $5 \mu \mathrm{m}$ average particle size. Column size was $250 \times 4 \mathrm{~mm}$ i.d. The cartridge temperature for the column was $32^{\circ} \mathrm{C}$.

Chromatograph 2: PSS SDV lin XL, $5 \mu \mathrm{m}$ average particle size and column size of $50 \times 20 \mathrm{~mm}$ i.d.

\section{Mobile Phase}

Chromatograph 1: (a) critical conditions for PS: tetrahydrofuran-acetonitrile 49:51 v/v; (b) critical conditions for PMMA: methyl ethyl ketone-cyclohexane 73.4:26.6 v/v. Chromatograph 2: tetrahydrofuran, all solvents were HPLC grade.

\section{Flow rates}

Chromatograph 1: off-line mode $1 \mathrm{~mL} / \mathrm{min}$, on-line mode $0.04 \mathrm{~mL} / \mathrm{min}$. Chromatograph 2: off-line mode $1 \mathrm{~mL} / \mathrm{min}$, on-line mode $4 \mathrm{~mL} / \mathrm{min}$.

\section{Detectors}

Waters 486 tunable UV detector at $254 \mathrm{~nm}$ and evaporative light scattering detector (ELSD) model 1000 of Polymer Laboratories both after chromatograph 2.

\section{Samples}

The block copolymers under investigation were from different sources. Sample 1 was prepared by Y. Gallot (Institute Charles Sadron, Strasbourg, France) through anionic polymerization using phenylisopropyl potassium as the initiator. Samples 2 and $\mathbf{3}$ were products of Polymer Standards Service $\mathrm{GmbH}$, Mainz, Germany. They were prepared by anionic polymerization using butyl lithium as the initiator. Samples 4 and $\mathbf{5}$ were experimental products of DKI, prepared by anionic polymerization using butyl lithium as the initiator. Samples $\mathbf{6}, \mathbf{6 a}, \mathbf{7}$, and $7 \mathbf{a}$ were prepared by E. Rizzardo (CSIRO Molecular Science, Melbourne, Australia) through RAFT. Tab. 3 summarizes the analytical data on molar masses and chemical composition that have been provided by the producers of the samples.

The critical points were adjusted using poly(methyl methacrylate) and polystyrene narrow disperse calibration standards of Polymer Standards Service $\mathrm{GmbH}$, Mainz, Germany. The same standards were used for the calibration of the SEC system.

Acknowledgement: The authors would like to express their gratitude to Y. Gallot (Institute Charles Sadron, Strasbourg, France), Polymer Standards Service GmbH (Mainz, Germany), and Ezio Rizzardo (CSIRO Molecular Science, Melbourne, Australia) for providing the samples. 
Tab. 3. Molar masses and chemical compositions (weight ratios PS:PMMA) of the PS-b-PMMA block copolymers as provided by the producers

\begin{tabular}{|c|c|r|r|c|}
\hline Sample & Type & $\mathrm{M}_{\mathrm{w}}$ in $\mathrm{g} / \mathrm{mol}$ & Composition & Source \\
\hline $\mathbf{1}$ & PS-b-PMMA & 180000 & $30: 70$ & Y. Gallot \\
$\mathbf{2}$ & PS-b-PMMA & 25000 & $50: 50$ & PSS \\
$\mathbf{3}$ & PS-b-PMMA & 108000 & $50: 50$ & PSS \\
& & (PS: 54000$)$ & & \\
$\mathbf{4}$ & PS-b-PMMA & 67000 & $50: 50$ & DKI \\
& & & & \\
$\mathbf{5}$ & PS-b-PMMA & 1106000 & $75: 25$ & DKI \\
$\mathbf{6}$ & PMMA-b-PS & 33000 & $40: 60$ & E. Rizzardo \\
$\mathbf{6 a}$ & PMMA & 20000 & $0: 100$ & E. Rizzardo \\
$\mathbf{7}$ & PMMA-b-PS & 63000 & $40: 60$ & E. Rizzardo \\
$\mathbf{7 a}$ & PMMA & 38000 & $0: 100$ & E. Rizzardo \\
\hline
\end{tabular}

[1] Pasch, H.; Trathnigg, B.; "HPLC of Polymers", Springer, Berlin-Heidelberg-New York 1998.

[2] Pasch, H.; Much, H.; Schulz, G.; Gorshkov, A.V.; LC-GC Internat. 1992, 5, 38.

[3] Gankina, E.; Belenkii, B.; Malakhova, I.; J. Planar Chromatogr. 1991, 4, 191.

[4] Zimina, T.M.; Kever, J.J.; Melenevskaya, E.Y.; Fell, A.F.; J. Chromatogr. 1992, 593, 233.

[5] Pasch, H.; Brinkmann, C.; Gallot, Y.; Polymer 1993, 34, 4100.

[6] Pasch, H.; Gallot, Y.; Trathnigg, B.; Polymer 1993, 34, 4986.

[7] Pasch, H.; Brinkmann, C.; Much, H.; Just, U.; J. Chromatogr. 1992, 623, 315.

[8] Gorbunov, A.A.; Skvortsov, A.M.; Vysokomol. Soedin. (A) 1988, 30, 453.

[9] Gorbunov, A.A.; Skvortsov, A.M.; Vysokomol. Soedin. (A) 1988, 30, 895.

[10] Entelis, S.G.; Evreinov, V.V.; Gorshkov, A.V.; Adv. Polym. Sci. 1986, 76, 129.

[11] Pasch, H.; Adv. Polym. Sci. 1997, 128, 1.

[12] Pasch, H.; Adv. Polym. Sci. 2000, 150, 1.

[13] Kilz, P.; Labor Praxis 1993, 6, 64. 
[14] Kilz, P.; Krüger, R.P.; Much, H.; Schulz, G.; ACS Adv. Chem. 1995, 247, 223.

[15] Kilz, P.; Krüger, R.P.; Much, H.; Schulz, G.; PMSE Preprints 1993, 69, 114.

[16] Trathnigg, B.; Maier, B.; Yan, X.; Proc. 7th Int. Symp. Polym. Anal. Char., Les Diablerets, Switzerland 1994.

[17] Adrian, J.; Braun, D.; Pasch, H.; LC-GC Int. 1998, 11, 32.

[18] (a) Murphy, R.E.; Schure, M.R.; Mink, L.P.; Foley, J.P.; Proc. Int. Symp. Polym. Anal. Char., A7, Sanibel Island, FL 1995. (b) Murphy, R.E.; Schure, M.R.; Foley, J.P.; Anal. Chem. 1998, 70, 1585.

[19] Krüger, R.P.; Much, H.; Schulz, G.; Int. J. Polym. Anal. Char. 1996, 2, 221.

[20] Adrian J.; Braun, D.; Pasch, H.; Angew. Makromol. Chem. 1999, 267, 82.

[21] Adrian, J.; Esser, E.; Hellmann, G.; Pasch, H.; Polymer 2000, 41, 2439.

[22] Augenstein, M.; Stickler, M.; Makromol. Chem. 1990, 191, 415.

[23] Siewing, A.; Schierholz, J.; Braun, D.; Hellmann, G.; Pasch, H.; Macromol. Chem. Phys. 2001, 202, 2890.

[24] Kilz P.; Pasch H.; "Coupled Liquid Chromatographic Techniques in Molecular Characterization", in Encyclopedia of Analytical Chemistry; Meyers, R.A., editor; J. Wiley \& Sons, Chichester 2001. 